\title{
Differential expression of immediate early genes Zif268 and c-Fos in the hippocampus and prefrontal cortex following spatial learning and glutamate receptor antagonism
}

\author{
Francesca R. Farina $^{\mathrm{a}, *}$, Sean Commins ${ }^{\mathrm{b}}$ \\ a School of Psychology, University College Dublin, Belfield, Dublin 4, Ireland \\ ${ }^{\mathrm{b}}$ Department of Psychology, Maynooth University, Maynooth, Co. Kildare, Ireland
}

\section{H I G H L I G H T S}

- NMDAR blockade alters basal levels of c-Fos expression in a region specific manner.

- Inhibition of NMDARs, but not AMPARs, disrupts spatial memory encoding.

- Inhibition of NMDARs attenuates expression of Zif268 but not c-Fos in area CA1.

- AMPAR blockade has no effect on spatial memory acquisition or IEG expression.

- Zif268 and c-Fos expression are not coordinated during spatial memory formation.

\section{A R T I C L E I N F O}

\section{Article history:}

Received 11 January 2016

Received in revised form 29 March 2016

Accepted 2 April 2016

Available online 9 April 2016

\section{Keywords:}

Glutamate receptors

Immediate early genes

Hippocampus

Prefrontal cortex

Spatial memory

\begin{abstract}
A B S T R A C T
The objective of this study was to examine the effects of NMDAR and AMPAR antagonism on the expression of Zif268 and c-Fos in the hippocampus and medial prefrontal cortex during spatial memory encoding in rats trained in the Morris water maze. NMDAR inhibition impaired navigation and significantly attenuated expression of Zif268, but not c-Fos, in area CA1. AMPAR channel blockade had little effect on learning or IEG expression. Overall, Zif268 and c-Fos displayed markedly different patterns of hippocampal and prefrontal expression, with Zif268 being more closely linked to spatial learning.
\end{abstract}

(c) 2016 Elsevier B.V. All rights reserved.
It is widely accepted that the formation of new spatial memories requires the hippocampus and activation of glutamate receptors therein [1]. The $N$-methyl-D-aspartate receptor (NMDAR) sub-class is thought to be particularly important, with studies showing that NMDAR blockade impairs performance in a range of spatial tasks [2]. Immediate early genes (IEGs) have also been implicated in spatial memory processing. IEGs are rapidly and transiently induced by neuronal activation, and accordingly, can be used as neuronal activity markers [3]. As expected, increases in hippocampal IEG expression (Zif268, c-Fos and Arc) have been reliably documented following spatial learning [4]. Further to their role as activity markers, IEGs also play a functional role in facilitating the cell modifications which underlie synaptic plasticity and, in turn, long-

\footnotetext{
* Corresponding author.

E-mail address: francesca.farina@ucd.ie (F.R. Farina).
}

term memory formation [5]. Their functional significance for spatial learning and memory has been demonstrated previously in studies showing that genetic ablation of IEGs leads to disrupted encoding and retrieval [3].

Despite the importance of both glutamate receptor and IEG activation for spatial learning, the way in which these processes interact during the memory formation has received little attention. To date, only one study has examined glutamate-IEG interactions in this context [6]. Czerniawski and colleagues [6] illustrated that antagonism of hippocampal NMDARs prior to contextual fear conditioning resulted in a significant attenuation of Arc expression in this region compared to naïve controls, suggestive of a functional coupling between NMDARs and Arc expression. However, a similar finding has yet to be reported for spatial memory. The objective of this paper was therefore to delineate the effects of glutamate receptor antagonism on the encoding of spatial memories and associated IEG expression. As administration of glutamate 
receptor antagonists has been shown to influence basal IEG expression [7], we examined their impact on IEG expression both before and after spatial training (Experiments 1 and 2). In addition to hippocampal areas CA1, CA3 and the dentate gyrus, we analysed IEG expression in the medial prefrontal cortex, due to its known role goal-directed behaviour [8]. A final aim of this study was to determine whether or not the expression of IEGs is coordinated during spatial learning. Current evidence is equivocal, with some studies reporting similar patterns of expression across multiple IEGs in the hippocampus [9], and others finding divergent activation patterns in the hippocampus and prefrontal cortex [10,11]. Thus, we charted pre- and post-learning expression of two commonly used IEGs: Zif268 and c-Fos.

Subjects were forty-seven male Wistar rats (three months old, 250-300 g) obtained from Charles River Laboratories, UK. Animals were housed three per cage in a temperature controlled environment $\left(\sim 21^{\circ} \mathrm{C}\right)$, had ad libitum access to water and food pellets and were maintained under a 12:12 h light:dark cycle (07:00-19:00). All experimentation was carried out during the light phase and animals were well handled and acclimatised to the testing environment beforehand. Guidelines for the maintenance and experimentation of animals conformed to the Department of Health and Children (Ireland) guidelines under statutory instrument (S.I.) No. 543 of 2012 and the European directive 2010/63/EU.

In Experiment 1, the influence of glutamate receptor blockade on basal expression of Zif268 and c-Fos in the hippocampus and prefrontal cortex was examined. Rats were randomly divided into three groups ( $n=5$ per group): MK-801, CNQX and Saline. MK-801 and CNQX groups were administered intraperitoneally (i.p.) with NMDAR antagonist MK-801 (0.1 mg/kg; Sigma-Aldrich) or $\alpha$-amino-3-hydroxy-5-methyl-4-isoxazolepropionic acid receptor (AMPAR) channel blocker CNQX ( $1.5 \mathrm{mg} / \mathrm{kg}$; Tocris Bioscience), respectively [12]. The Saline group was administered with physiological saline $(0.1 \mathrm{ml} / 100 \mathrm{~g}$ body weight of $0.9 \%$ sodium chloride; Sigma, Ireland). All subjects received one injection per day for five days (matched to spatial training in Experiment 2). After drug administration, animals were returned to their home cages and monitored for any drug-induced locomotive behaviours.

Ninety minutes post-injection on day five, animals were terminally anaesthetised with sodium pentobarbital $(60 \mathrm{mg} / \mathrm{kg}$, Euthatal) and perfused transcardially with $0.9 \%$ phosphate buffered saline (PBS, $250 \mathrm{ml}, \mathrm{Ph} 7.4$ ), followed by $4 \%$ paraformaldehyde in $0.1 \mathrm{M}$ phosphate buffer ( $\mathrm{PB}, 300 \mathrm{ml}, \mathrm{Ph} 7.4$ ). Brains were removed and post-fixed in $4 \%$ paraformaldehyde overnight at $4{ }^{\circ} \mathrm{C}$ before being cryoprotected in $30 \%$ sucrose solution. Coronal sections (40$\mu \mathrm{m}$-thick; see Fig. $1 \mathrm{~A}$ for distance from Bregma) were cut using a freezing stage sledge microtome (Brights Instruments, Huntingdon, $\mathrm{UK})$, and free floating sections were stored in $0.1 \mathrm{M}$ PB containing $0.01 \%$ sodium azide $\left(4^{\circ} \mathrm{C}\right)$. To minimise variation in staining specificity due to the immunohistochemical procedure, brain sections representing each region of interest from all animals were processed side-by-side in a single batch. Immunohistochemistry followed a standard Avidin-Biotin Complex/Nickel DAB colorimetric protocol [13]. Zif268 and c-Fos were labelled using Zif268/Egr-1, rabbit polyclonal antibody (dilution 1:3000; Santa Cruz Biotechnology), and c-Fos, rabbit polyclonal antibody (dilution 1:2000; Santa Cruz Biotechnology), respectively. Reaction times were standardised for all sections to ensure comparable staining intensity across groups. Sections were mounted onto gelatin-coated slides, dehydrated, cleared in Histoclear (National Diagnostics, Hull, UK), and coverslipped using Eukitt (Sigma, Poole, UK).

Images of dorsal hippocampal (CA1, CA3, dentate gyrus) and medial prefrontal regions were taken using an Olympus digital camera (Camedia C-2020-Z) mounted on an Olympus BX-50 microscope ( $4 \times$ magnification; see Fig. 1 for exact areas sampled). To eliminate experimenter bias in the cell counting process, counts were automatically calculated by the public domain program Image (National Institute of Health, USA) as per Ref. [14]. Specifically, cells above a pre-defined brightness intensity threshold of 100 , and within a pre-defined particle area size (20-200 pixel range) were included. Counts from four sections per animal were calculated for all regions. These counts were averaged to produce individual subject means per region. Subject means were then averaged according to group to produce group means. As all immunohistochemical procedures were carried out in a single session, normalisation of the data was not necessary and raw scores were used in statistical analyses [10]. Statistical analyses were carried out using SPSS (Version 22). IEG expression across groups was compared using one-way ANOVA with Tukey post hoc tests.

No significant group differences in basal Zif268 expression were noted for any area investigated (see Figs. 1 B, 2 A ). For c-Fos expression, no significant differences were found in hippocampal sub-regions; however, a significant main effect of group was recorded in the medial prefrontal cortex $\left(F_{2,14}=8.98, P=0.05\right)$, where c-Fos expression was significantly elevated for the MK-801 group relative to the Saline and CNQX groups (both $P<0.01$; see Figs. 1 C, 2 B).

Once the baseline effects of glutamate receptor blockade on IEG expression had been established, we examined its influence on spatial memory acquisition and on associated Zif268 and c-Fos expression in the hippocampus and prefrontal cortex. In Experiment 2, experimentally naive animals were randomly allocated to one of four groups; Saline, MK-801, CNQX and Control ( $n=8$ per group). Saline, MK-801 and CNQX groups were spatially trained in the hidden platform version of the Morris water maze [16] over five days (four trials per day; see Fig. 3A). The experimental set up, including the maze, platform, and distal cues (two light bulbs), were identical to those described previously in Farina et al. [15]. Trials were observed by the experimenter in an adjacent testing room via a video camera positioned directly above the centre of the maze. Behavioural data were recorded using EthoVision ${ }^{\odot}$ tracking system (Noldus Information Technologies, Wageningen, Netherlands).

Twenty minutes prior to training each day, rats received an i.p. injection of saline $(0.1 \mathrm{ml} / 100 \mathrm{~g}$ body weight of $0.9 \% \mathrm{NaCl})$, MK$801(0.1 \mathrm{mg} / \mathrm{kg})$ or CNQX (1.5 mg/kg) [12]. Saline-treated animals acted as a trained comparison group, allowing us to contrast IEG expression across treatment conditions while controlling for spatial task demands. Spatial training was carried out over five days as described previously in Farina et al. [17]. The Control group received no training; they remained in their cages for five days, receiving one i.p. saline injection per day $(0.1 \mathrm{ml} / 100 \mathrm{~g}$ body weight of $0.9 \% \mathrm{NaCl}$ ). This group provided a baseline measure of IEG expression. Ninety minutes post-injection on day five, all animals were terminally anaesthetised, perfused transcardially, and their brains were removed, post-fixed and cryoprotected as per Experiment 1. Immunohistochemical staining was performed for all groups in a single session, thus cell counts were not normalised.

Behavioural task acquisition was assessed using escape latency (in seconds). Daily escape latencies were averaged for each animal, which were then averaged according to group to yield daily group means. Escape latencies were analysed using a $3 \times 5$ mixed factorial ANOVA (within-groups measure: day; between-groups measure: group). Analyses of individual group escape latencies were examined using one-way repeated-measures ANOVA with Bonferroni correction. Analyses of group differences on individual training days were assessed using one-way between-groups ANOVA with Tukey post hoc tests. Zif268 and c-Fos immunopositive cell counts in the hippocampus and medial prefrontal cortex were automatically counted and averaged as per Experiment 1. Regional group differences in Zif268 and c-Fos expression were investigated using one-way between-groups ANOVAs with Tukey post hoc compar- 
A

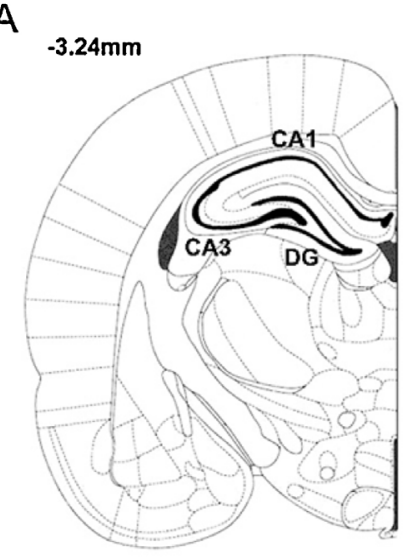

C

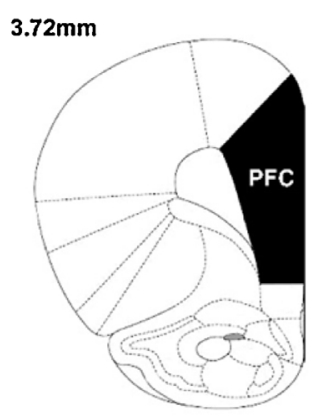

B
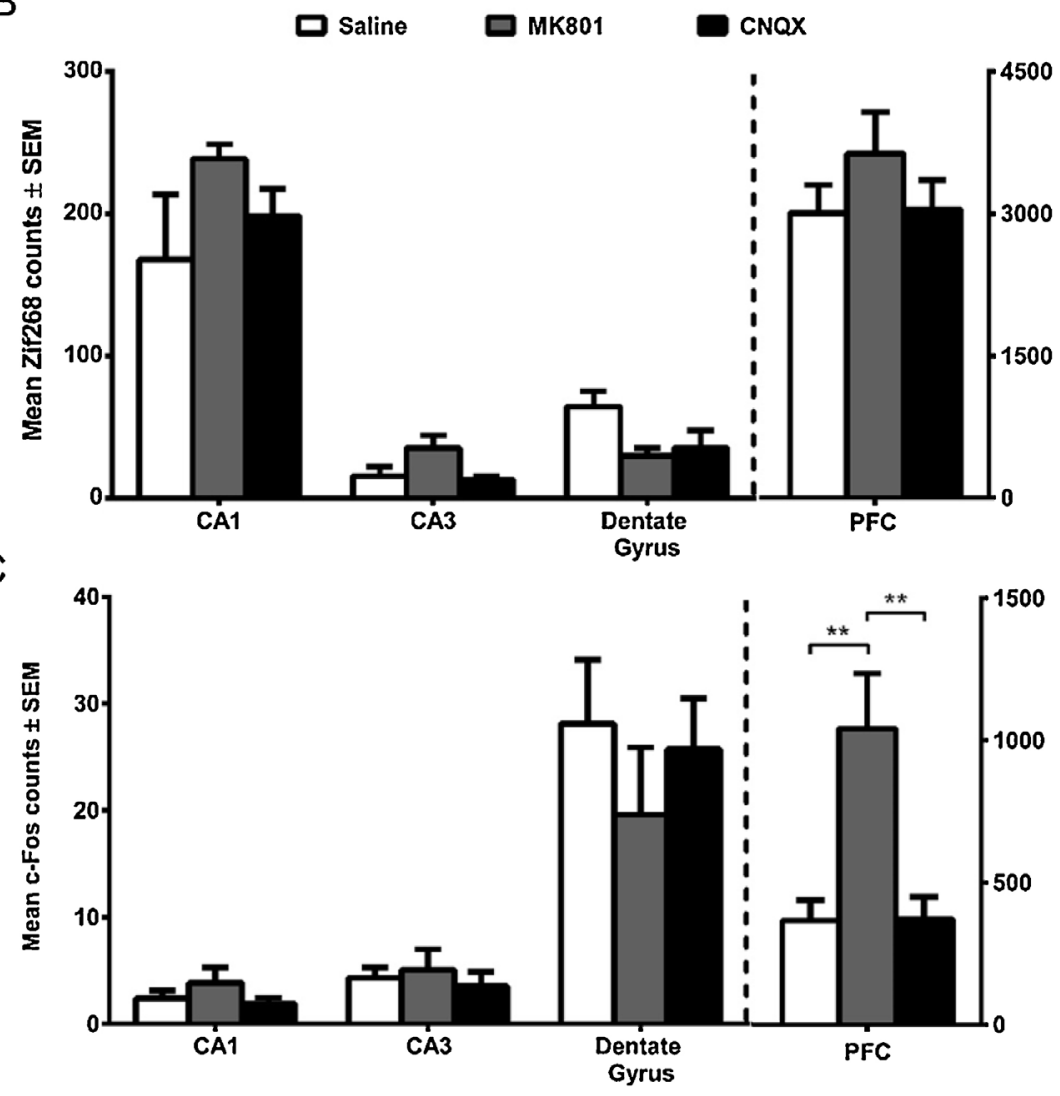

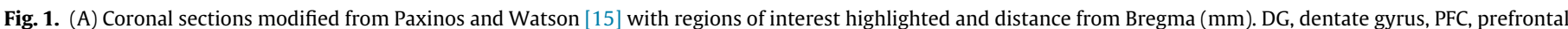

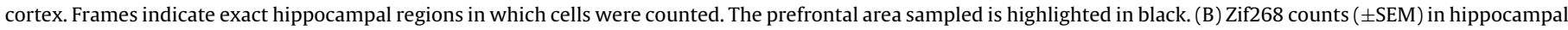

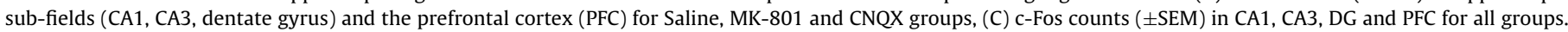

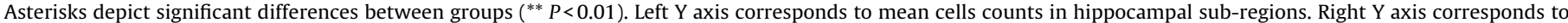
mean cell counts in the prefrontal cortex.

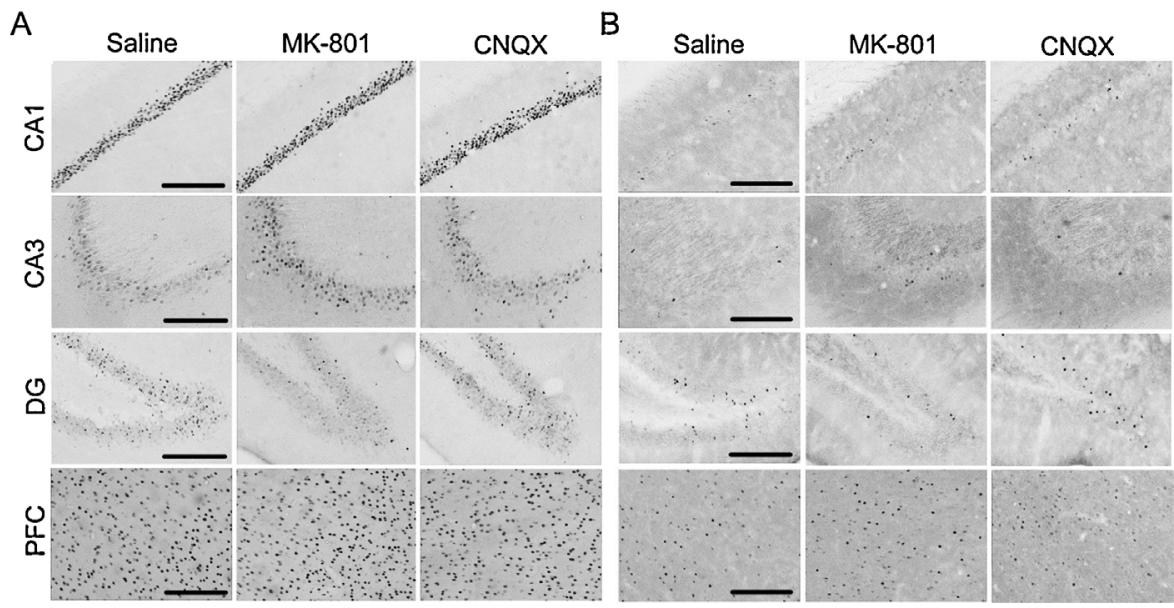

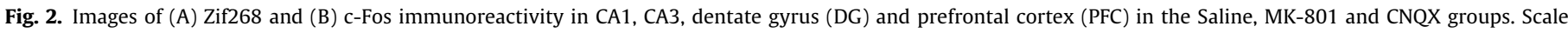
$\mathrm{bar}=100 \mu \mathrm{m}$. Images are cropped from the total area sampled for ease of viewing.

isons (for trained groups) and independent samples $t$ tests (trained versus non-trained animals; corrected for multiple comparisons).

Significant main effects of day $\left(F_{4,84}=15.55, P=0.001\right.$, partial eta $\left.^{2}=0.43\right)$ and group $\left(F_{2,21}=50.42, P=0.001\right.$, partial eta $\left.{ }^{2}=0.83\right)$ were found. The day $\mathrm{x}$ group interaction was not significant. Bonferroni post hoc comparisons showed that mean escape latency on day 5 was significantly faster than on day $1(P=0.001)$. Tukey post hoc tests showed that the MK-801 group was significantly slower at escaping the maze compared to both Saline and CNQX groups (both $P=0.001$; see Fig. $3 \mathrm{~A}$ ). Individual main effects of day were also noted for the Saline $\left(F_{4,28}=17.02, P=0.001\right.$, partial eta $\left.{ }^{2}=0.71\right)$ and CNQX groups $\left(F_{4,28}=6.22, P=0.001\right.$, partial eta $\left.{ }^{2}=0.47\right)$. Bonferroni post hoc tests showed that Saline-treated animals escaped the maze faster on day 5 relative to day $1(P=0.02)$. Post hoc tests were nonsignificant for the CNQX group. No main effect of day was observed for the MK-801 group $\left(F_{4,28}=2.35, P=0.08\right.$, partial eta $\left.{ }^{2}=0.25\right)$. The 
A
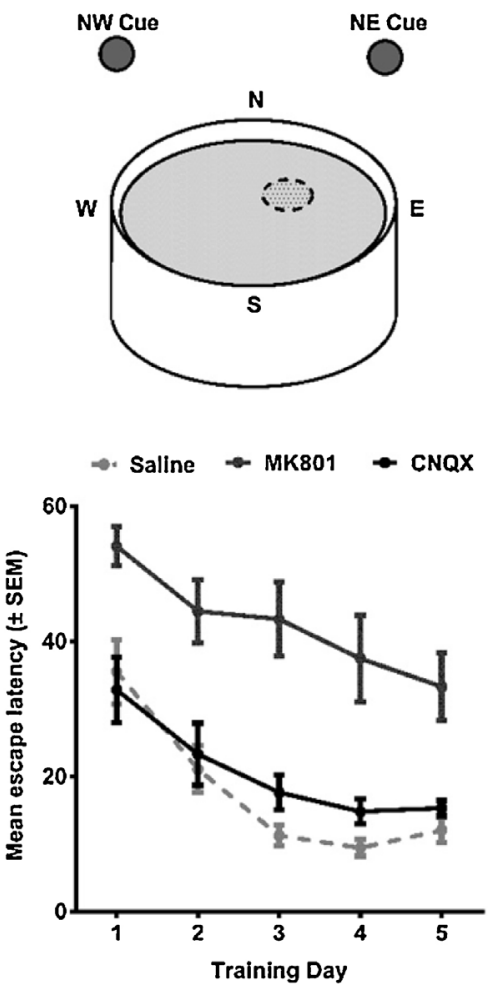

B

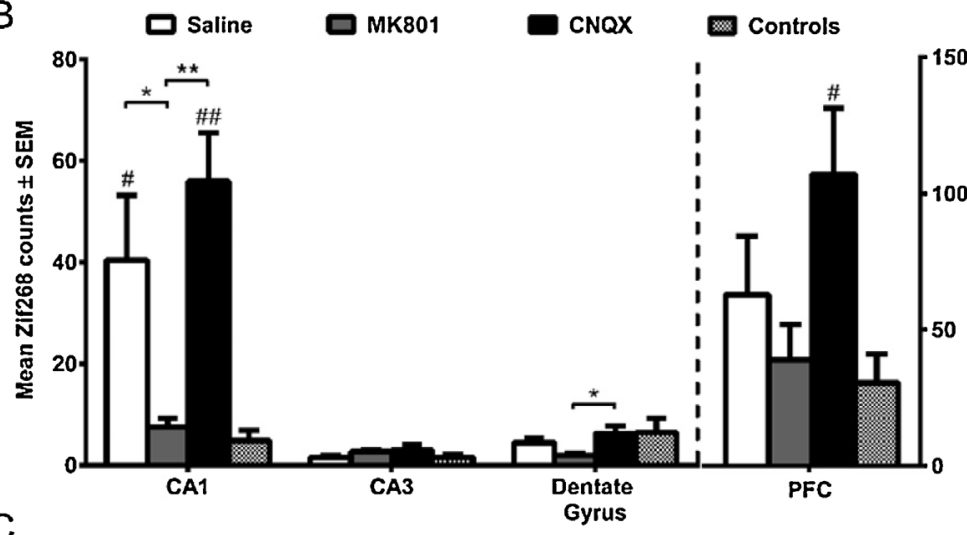

C

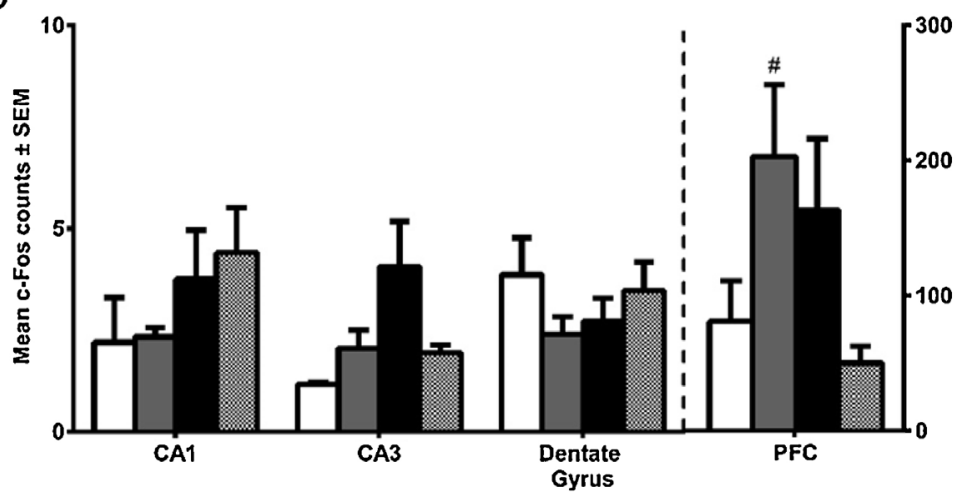

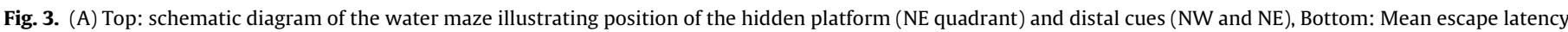

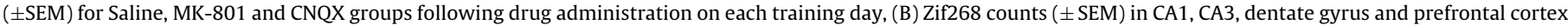

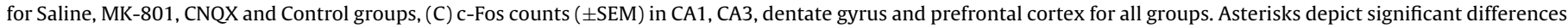

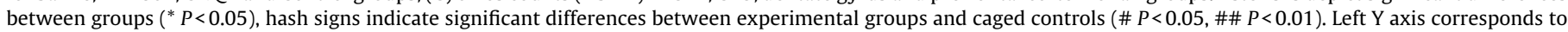
mean cells counts in hippocampal sub-regions. Right $Y$ axis corresponds to mean cell counts in the prefrontal cortex.

MK-801 group were also significantly slower at locating the platform compared to the other groups on all days (day $1: F_{2,23}=7.40$, $P=0.01$, day $2: F_{2,23}=9.02, P=0.01$, day $3: F_{2,23}=22.57, P=0.001$, day 4: $F_{2,23}=14.51, P=0.001$, and day $5: F_{2,23}=13.00, P=0.001$; Tukey post hoc tests: all $P<0.03$ ).

When Zif268 expression levels in trained animals were compared, main effects of group were found in CA1 $\left(F_{2,23}=7.16, P=0.01\right)$ and the dentate gyrus $\left(F_{2,23}=5.00, P=0.02\right)$. CA1 Zif268 counts were significantly higher for Saline and CNQX groups compared to the MK-801 group (Tukey: $P=0.05$ and $P=0.01$, respectively; see Figs. $3 \mathrm{~B}, 4 \mathrm{~A}$ ). Dentate gyrus Zif268 expression was greater for the CNQX group relative to the MK-801 group (Tukey: $P=0.02$ ). Zif268 counts were also significantly elevated from baseline (i.e. untrained controls) for Saline and CNQX groups in area CA1 $\left(t_{14}=2.77\right.$, $P=0.05$, and $\left.t_{14}=5.28, P=0.01\right)$, and for the CNQX group in the prefrontal cortex $\left(t_{14}=2.91, P=0.03\right.$; see Fig. $\left.3 \mathrm{~B}\right)$. No group differences in hippocampal c-Fos levels were found, while prefrontal c-Fos expression for the MK-801 group was significantly higher than baseline $\left(t_{14}=2.78, P=0.04\right.$; see Figs $\left.3 \mathrm{C}, 4 \mathrm{~B}\right)$.

In this study we characterised the effects of NMDAR and AMPAR antagonism on the expression of Zif268 and c-Fos in the hippocampus and medial prefrontal cortex before and after spatial training in the Morris water maze. NMDAR blockade led to a marked increase in basal prefrontal c-Fos (but not Zif268) expression, thereby demonstrating that, even at a relatively low dose, NMDA antagonism can significantly influence gene expression in the absence of any spatial training. However, hippocampal IEG expression was unaffected, suggesting that NMDAR blockade alters baseline expression of c-Fos in a region specific manner. These findings have important implications for IEG imaging research involving pharmacological interventions, particularly with regard to c-Fos as a regional marker of neuronal activity. In contrast to NMDARs, inhibition of AMPARs had no effect on basal IEG expression in any area analysed.

Antagonism of NMDARs during spatial training resulted in dramatically impaired learning. The MK- 801 group failed to improve at finding the platform over the course of training, and took considerably longer to reach the target compared to saline- and CNQX-treated animals. While MK-801 administration has previously been shown to disrupt motor functioning [18], we observed no such effects; thus, the poor performance of the MK-801 group cannot be attributed to a movement deficit. Instead, our results support the suggestion that NMDAR activation is important for spatial memory performance [19], even after multiple days of training. In addition to impaired navigation, NMDAR blockade led to a significant reduction in Zif268 (but not c-Fos) expression in CA1, relative to rats treated with saline or CNQX. Moreover, mean Zif268 expression for the MK-801 group in this area was comparable to that of the cage control group; although it should be noted that the suitability of cage controls as a comparison group continues to be debated [10]. Together, results indicate a strong association between NDMAR activation and Zif268 expression in CA1 during spatial learning, which likely reflects their underlying roles in learning-related plasticity. Again, AMPAR channel blockade had little effect on IEG expression, and animals in this group successfully acquired the water maze task. These results are in keeping with the proposal that AMPARs are more closely linked to memory retrieval [19].

Our results highlight the importance of area CA1 for spatial memory, and support the current consensus that this region is crucial for creating spatial representations of the environment [1]. Greater Zif268 activation in the medial prefrontal cortex was also 
A

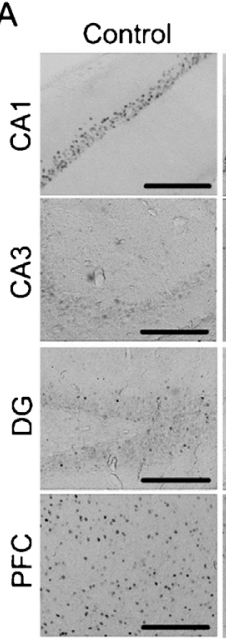

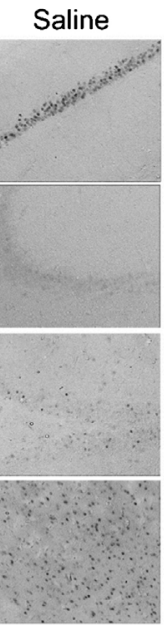
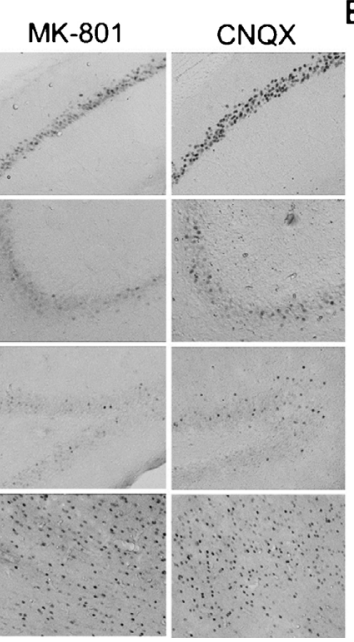

B
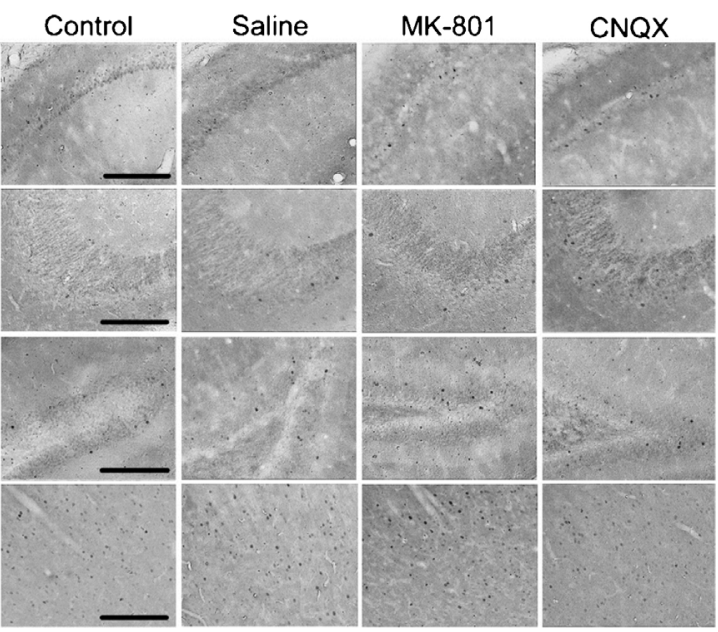

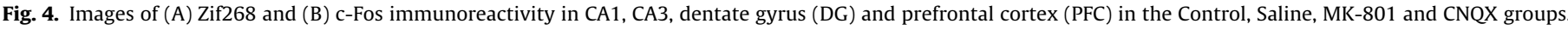
Scale bar $=100 \mu \mathrm{m}$. Images are cropped from the total area sampled for ease of viewing.

associated with animals that learned the task, implying a link with spatial learning. This would not be surprising given the known connections between this region and area CA1 [8]. However, because the difference between saline-treated rats and cage controls failed to reach statistical significance, it is difficult to interpret the degree of prefrontal involvement. Conversely, area CA3 and the dentate gyrus did not appear to be engaged by the water maze task, with only one small group difference in Zif268 expression being found in the latter region. One possible explanation for this is the environmental set up used. More specifically, CA3 and the dentate gyrus are thought to be particularly important for pattern completion and separation, respectively [20], neither of which were expected here as our environment was fixed throughout training.

In contrast to previous research (see Ref. [4]), hippocampal cFos expression did not differ between groups that learned the task and those that did not. We suggest that this is due to the longer length of training employed relative to other studies (typically one to three days of training). Thus, c-Fos expression levels may have peaked earlier during the encoding process, and were returning to baseline by day five [21]. Moreover, the sustained increase in prefrontal c-Fos expression was likely due to NMDAR antagonism, as per the baseline results. Finally, our data illustrate that Zif268 and c-Fos expression is not coordinated in the hippocampus or prefrontal cortex in the context of spatial reference memory. These results are comparable to those of Shires and Aggleton [10], who also found different patterns of Zif268 and c-Fos expression in the hippocampus and prefrontal cortex in rats after extensive training in a spatial working memory task. These findings are particularly salient, given that IEGs are often used interchangeably as markers of neuronal activation. We therefore propose that Zif268 represents a more robust index of spatial learning in the Morris water maze task.

\section{Acknowledgement}

This work was funded by the Maynooth University Doctoral Teaching scholarship held by F.R.F.

\section{References}

[1] K. Nakazawa, et al., NMDA receptors: place cells and hippocampal spatial memory, Nat. Rev. Neurosci. 5 (5) (2004) 361-372.

[2] I. Lee, R.P. Kesner, Differential contribution of NMDA receptors in hippocampal subregions to spatial working memory, Nat. Neurosci. 5 (2) (2002) 162-168.
[3] S. Kubik, T. Miyashita, J.F. Guzowski, Using immediate-early genes to map hippocampal subregional functions, Learn. Mem. 14 (11) (2007) 758-770.

[4] D.N. Barry, S. Commins, Imaging spatial learning in the brain using immediate early genes: insights, opportunities and limitations, Rev. Neurosci. 22 (2) (2011) 131-142.

[5] W. Tischmeyer, R. Grimm, Activation of immediate early genes and memory formation, Cell. Mol. Life Sci. 55 (4) (1999) 564-574.

[6] J. Czerniawski, et al., The importance of having arc: expression of the immediate-early gene arc is required for hippocampus-dependent fear conditioning and blocked by NMDA receptor antagonism, J. Neurosci. 31 (31) (2011) 11200-11207.

[7] X.-M. Gao, T. Hashimoto, C.A. Tamminga, Phencyclidine (PCP) and dizocilpine (MK801) exert time-dependent effects on the expression of immediate early genes in rat brain, Synapse 29 (1) (1998) 14-28.

[8] J.C. Churchwell, et al., Prefrontal and hippocampal contributions to encoding and retrieval of spatial memory, Neurobiol. Learn Mem. 93 (3) (2010) 415-421.

[9] J.F. Guzowski, et al., Experience-dependent gene expression in the rat hippocampus after spatial learning: a comparison of the immediate-early genes Arc, c-fos, and zif268, J. Neurosci. 21 (14) (2001) 5089-5098.

[10] K.L. Shires, J.P. Aggleton, Mapping immediate-early gene activity in the rat after place learning in a water-maze: the importance of matched control conditions, Eur. J. Neurosci. 28 (5) (2008) 982-996.

[11] L.A. Teather, et al., Differential induction of c-Jun and Fos-like proteins in rat hippocampus and dorsal striatum after training in two water maze tasks, Neurobiol. Learn Mem. 84 (2) (2005) 75-84.

[12] J. Kealy, S. Commins, Antagonism of glutamate receptors in the CA1 to perirhinal cortex projection prevents long-term potentiation and attenuates levels of brain-derived neurotrophic factor, Brain Res. 1265 (2009) 53-64

[13] A.N. Coogan, H.D. Piggins, Circadian and photic regulation of phosphorylation of ERK1/2 and Elk-1 in the suprachiasmatic nuclei of the Syrian hamster, J. Neurosci. 23 (7) (2003) 3085-3093.

[14] D.N. Barry, A.N. Coogan, S. Commins, The time course of systems consolidation of spatial memory from recent to remote retention: a comparison of the immediate early genes Zif268, c-Fos and Arc, Neurobiol. Learn. Mem. 128 (2016) 46-55.

[15] G. Paxinos, C. Watson, The Rat Brain in Stereotaxic Coordinates-The New Coronal Set, Academic press, 2004

[16] R.G.M. Morris, Spatial localization does not require the presence of local cues, Learn. Motiv. 12 (2) (1981) 239-260.

[17] F.R. Farina, et al., Learning efficiency: the influence of cue salience during spatial navigation, Behav. Process. 116 (2015) 17-27.

[18] E.L. Hargreaves, D.P. Cain, Hyperactivity, hyper-reactivity, and sensorimotor deficits induced by low doses of the $N$-methyl-D-aspartate non-competitive channel blocker MK801, Behav Brain Res. 47 (1) (1992) 23-33.

[19] T. Bast, B.M. da Silva, R.G. Morris, Distinct contributions of hippocampal NMDA and AMPA receptors to encoding and retrieval of one-trial place memory, J. Neurosci. 25 (25) (2005) 5845-5856.

[20] M.A. Yassa, Z.M. Reagh, Competitive trace theory: a role for the hippocampus in contextual interference during retrieval, Front. Behav. Neurosci. 7 (2013) 107.

[21] J. He, K. Yamada, T. Nabeshima, A role of Fos expression in the CA3 region of the hippocampus in spatial memory formation in rats, Neuropsychopharmacology 26 (2) (2002) 259-268. 